\title{
New very massive stars in Cygnus OB2 ${ }^{\star}$
}

\author{
I. Negueruela ${ }^{1,2}$, A. Marco ${ }^{1,2}$, A. Herrero ${ }^{3,4}$, and J. S. Clark ${ }^{2}$ \\ 1 Departamento de Física, Ingeniería de Sistemas y Teoría de la Señal, Universidad de Alicante, Apdo. 99, 03080 Alicante, Spain \\ e-mail: ignacio@dfists.ua.es \\ 2 Department of Physics and Astronomy, The Open University, Walton Hall, Milton Keynes MK7 6AA, UK \\ 3 Instituto de Astrofísica de Canarias, 38200 La Laguna, Tenerife, Spain \\ 4 Departamento de Astrofísica, Universidad de La Laguna, Avda. Astrofísico Francisco Sánchez, s/n, 38071 La Laguna, Spain
}

Received 29 April 2008 / Accepted 9 June 2008

\section{ABSTRACT}

\begin{abstract}
Context. The compact association Cygnus OB2 is known to contain a large population of massive stars, but its total mass is currently a matter of debate. While recent surveys have uncovered large numbers of OB stars in the area around Cyg OB2, detailed study of the optically brightest among them suggests that most are not part of the association.

Aims. We observed an additional sample of optically faint $\mathrm{OB}$ star candidates, with the aim of checking if more obscured candidates are correspondingly more likely to be members of Cyg OB2.

Methods. Low resolution spectra of 9 objects allow the rejection of one foreground star and the selection of four O-type stars, which were later observed at higher resolution. In a subsequent run, we observed three more stars in the classification region and three other stars in the far red.

Results. We identify five (perhaps six) new evolved very massive stars and three main sequence O-type stars, all of which are likely to be members of Cyg OB2. The new findings allow a much better definition of the upper HR diagram, suggesting an age $2.5 \mathrm{Myr}$ for the association and hinting that the $\mathrm{O} 3-5$ supergiants in the association are blue stragglers, either younger or following a different evolutionary path from other cluster members. Though the bulk of the early stars seems to belong to an (approximately) single-age population, there is ample evidence for the presence of somewhat older stars at the same distance.

Conclusions. Our results suggest that, even though Cyg OB2 is unlikely to contain as many as 100 O-type stars, it is indeed substantially more massive than was thought prior to recent infrared surveys.
\end{abstract}

Key words. open clusters and associations: individual: Cyg OB2 - stars: formation - stars: luminosity function, mass function stars: early-type

\section{Introduction}

Among Galactic OB associations, Cyg OB2 is special in many respects. For a start, it is known to host a large population of massive stars, including a significant fraction of the earliest spectral types in the Galaxy (Walborn et al. 2002). The optical extinction to Cyg OB2 is high, but not sufficiently so that it prevents spectra of its stars in the classification region being taken (something impossible for other very massive open clusters with a large population of massive stars, such as Westerlund 1, Clark et al. 2005; or the Arches Cluster, Figer et al. 2002). Because of this, Cyg OB2 is a very useful laboratory, since, on one hand, it provides a large homogeneous population of OB stars that can be analysed (Herrero et al. 1999, 2002) and, on the other, can be used as a template to compare optical and infrared investigations (e.g., Hanson 2003). Finally, because of its compactness and high stellar content, Cyg OB2 seems to occupy a more or less unique position somewhat intermediate between an open cluster and a normal OB association (cf. Knödlseder 2000).

These properties have led to a great deal of interest in Cyg OB2, from the "classical" study of Johnson \& Morgan (1954) to the comprehensive investigation by Massey \& Thompson (1991), who identified $\sim 60$ stars more massive than $15 M_{\odot}$. More recently, based on star counts in the 2MASS observations of the region, Knödlseder (2000) proposed that the number of O-type stars in Cyg OB2 was much larger. Building on this

^ Figure 8 and Table 3 are only available in electronic form at http: //www . aanda. org result, Comerón et al. (2002) preselected a large number of possible OB members of Cyg OB2 from their 2MASS colours and obtained low-resolution $H$ - and $K$-band spectroscopy of the candidates. Candidates that lacked molecular bands were selected as very likely early-type stars. Of 77 candidates so selected, 31 stars for which optical spectra existed were OB stars, suggesting that most, if not all, of the other 46 objects were also OB stars in Cyg OB2.

From this list of candidates, Hanson (2003) selected those brightest in the optical (14 objects with $B=12$ to 14 ), for which she obtained classification spectra, finding that all of them were indeed OB stars. However, Hanson (2003) argues that most of these objects are not members of Cyg OB2. For a start, they all lie at some distance from the previously defined boundaries of Cyg OB2, as most of the sources located by Comerón et al. (2002) do. Moreover, about half of the objects observed are late $\mathrm{O}$ and early $\mathrm{B}$ supergiants, indicating ages rather larger than the 2 Myr that Hanson (2003) derives for Cyg OB2 from isochrone fitting to the location of the main sequence. Finally, one star (A39, B2 V) appears far too bright for its spectral type and is almost certainly a foreground object.

It is therefore an open question as to whether the list of candidates from Comerón et al. (2002) really contains a high fraction of actual Cyg OB2 members. Here we investigate this issue with new spectra of several other fainter optical candidates. We also make use of the recent publication of a large catalogue of accurate spectral types for Cyg OB2 members (Kiminki et al. 2007), which combined with our results and those of Hanson (2003), 
allows an enormous improvement in the characterisation of the HR diagram for the association.

In what follows, we will use the notation of Comerón et al. (2002) for stars within their list (A\#\# for OB candidates and

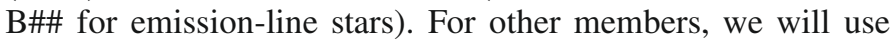
the numbering system of Massey \& Thompson (1991), with prefix MT, except for the twelve stars with the classical numbering of Johnson \& Morgan (1954), which are given with the symbol \# followed by their number.

\section{Observations}

Candidate stars from the list of Comerón et al. (2002) were observed with the 1.52-m Cassini telescope at the Loiano Observatory (Italy) during the nights of 2004 July 15-18. The telescope was equipped with the Bologna Faint Object Spectrograph and Camera (BFOSC) and an EEV camera. We used grism \#3, which covers 3300-5800 A with a resolution of $\sim 6 \AA$. Unfortunately, on the night of July 16th, the sky was very poor, with some veiling, and we resorted to observing two stars with the lower-resolution grism \#4. The night of July 17 th we could not observe. Therefore, in total, we observed only 10 stars, of which one, A27, had been observed before with better resolution and signal-to noise ratio (SNR) by Hanson (2003).

From these ten objects we selected five to be observed at higher resolution; the four which appeared to be O-type stars (based on the analysis presented in Sect. 3.1) and one that was probably a B-type dwarf as a check. These objects were observed with the 4.2-m William Herschel Telescope (WHT) in La Palma (Spain), equipped with the ISIS double-beam spectrograph, during a service run in June 2006. The instrument was fitted with the R300R grating and MARCONI2 CCD in the red arm and the R300B grating and EEV\#12 CCD in the blue arm. Both configurations result in a nominal dispersion of $0.85 \AA$ /pixel (the resolution element is approximately 3 pixels in the blue an 2 pixels in the red).

As the selection criteria of Comerón et al. (2002) proved sound, we then selected some objects from their list with very bright $K$ magnitudes (which should be intrinsically brightest) and observed them during a run on 2007 August 21, 22 at the WHT. Three objects were observed in the blue with grating R1200B (nominal dispersion of $\sim 0.23 \AA /$ pixel) and three others (whose $B>16$ made too faint for the blue grating) were observed with the red arm and grating R600R in the I-band, where relatively accurate classification is also possible (e.g., Clark et al. 2005). This configuration has a nominal dispersion of $\sim 0.5 \AA /$ pixel.

All the spectra have been reduced with the Starlink packages CCDPACK (Draper et al. 2000) and FIGARO (Shortridge et al. 1997) and analysed using FIGARO and DIPSO (Howarth et al. 1998).

\section{Results}

\subsection{Loiano spectra}

The Loiano spectra have rather poor SNR in the blue, but allow a rough classification of the stars. One of the candidates, A40, turns out to be a foreground G-type star. The other 9 objects are very obviously OB stars. Their spectra are displayed in Fig. 1, while their 2MASS magnitudes and derived spectral types are listed in Table 1.

A 11 has He I $4471 \AA \approx$ He II $4542 \AA$, no visible He II $4686 \AA$ (at this resolution; we see it in the WHT spectrum) and

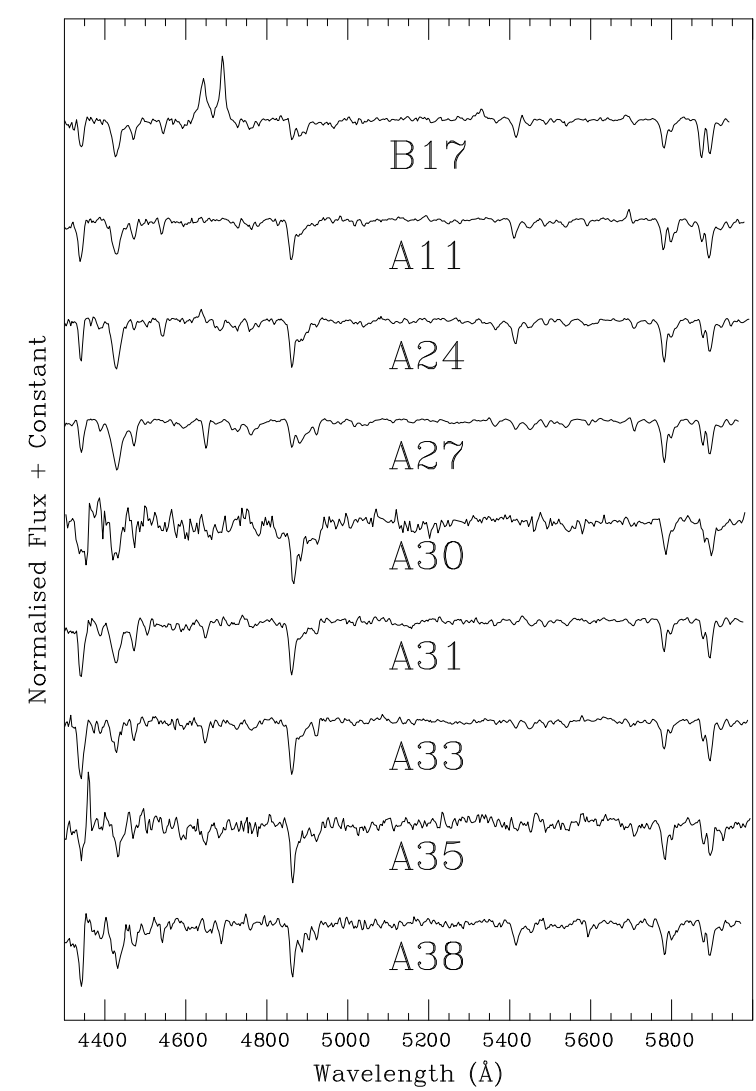

Fig. 1. Useful section of the spectra obtained from Loiano. The SNR decreases quickly towards the blue and there are essentially no counts bluewards of $\sim 4200 \AA$. The spectrum of A40, which turned out to be a foreground star, is not shown.

Table 1. Infrared 2MASS photometry and derived spectral types for programme stars.

\begin{tabular}{lcccc}
\hline \hline Name & $\left(J-K_{\mathrm{S}}\right)$ & $K_{\mathrm{S}}$ & Spectral type & Telescope \\
\hline A11 & 1.19 & 6.64 & O7.5 Ib-II(f) & L, WHT1 \\
A12 & 1.21 & 5.72 & B0 Ia & L, WHT2 \\
A15 & 1.14 & 6.81 & O7 Ib(f) & L,WHT2 \\
A18 & 1.07 & 8.35 & $\sim$ O8 V & WHT3 \\
A24 & 0.97 & 7.46 & O6.5 III((f)) & L,WHT1 \\
A25 & 1.01 & 7.36 & $\sim$ O8 III & WHT3 \\
A26 & 0.97 & 8.19 & O9.5 V & L, WHT2 \\
A27 & 0.97 & 5.75 & $\sim$ B0 I & L \\
A30 & 0.81 & 8.61 & $\sim$ B2 V & L \\
A31 & 0.95 & 7.98 & $\sim$ B0.5 V & L \\
A33 & 0.87 & 8.60 & B0.2 V & L, WHT1 \\
A35 & 0.81 & 8.47 & $\sim$ B0 V & L \\
A38 & 0.85 & 8.56 & O8 V & L, WHT1 \\
\hline B10 & 1.45 & 8.12 & Be & WHT3 \\
B17 & 1.21 & 6.44 & Ofpe & L,WHT1,WHT3 \\
\hline
\end{tabular}

${ }^{a}$ B0 Ia (Hanson 2003).

Key for telescope configurations: L - Loiano Cassini Telescope, WHT1 - WHT in 2006 with blue arm, WHT2 - WHT in 2007 with blue arm, WHT3 - WHT in 2007 with red arm (I-band only).

C III $5696 \AA$ strongly in emission. It is thus an $\sim 07$ supergiant. A24 has He I $4471 \AA$ < He II $4542 \AA$ and He II $4686 \AA$ in absorption and so it is a relatively unevolved mid O-type star. A27 has very prominent C III 4650 Å no He II 4686 Å, weak He II $4512 \AA$, strong $\mathrm{He}$ I lines and very weakened $\mathrm{H} \beta$. It should be $\mathrm{a} \sim \mathrm{B} 0$ supergiant, and indeed it has been classified as BO Ia by Hanson (2003), based on higher quality spectra. 


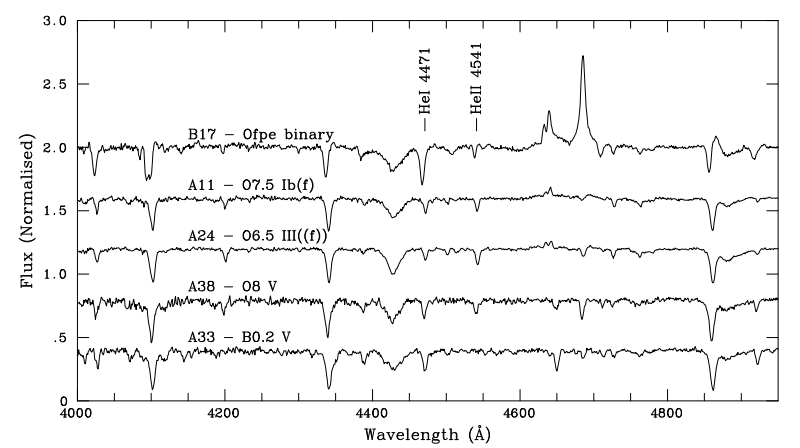

Fig. 2. Classification spectra of the 5 objects observed with ISIS on the WHT in June 2006.

The spectrum of A30 has lower resolution and SNR than the rest, but extends into the red. The lack of He II $4512 \AA$ makes it later than $\mathrm{B} 0$, while the fact that $\mathrm{H} \alpha$ is much deeper than the He I 6678, $7065 \AA$ lines suggests that it is a mid B star (e.g., later than $\sim \mathrm{B} 2 \mathrm{~V})$.

A31 has moderately strong C III $4650 \AA$ and very weak He II $4686 \AA$ and He II $4512 \AA$, suggesting a main sequence star in the B0-1 range. A33 is similar, with a slightly stronger C III $4650 \AA$, perhaps suggesting a higher luminosity. A35 has stronger He II $4686 \AA$, but is unlikely to be much earlier, as He II $4512 \AA$ is weak.

A38 has moderately strong He II lines, but He I $4471 \AA$ > He II $4542 \AA$, suggesting a late O-type star, while the lack of emission lines indicates a low luminosity. Finally B17 is characterised by strong emission lines of He II $4686 \AA$ and N III, and may be an extreme Of supergiant or an Ofpe/WNL star.

\subsection{WHT spectra}

Figure 2 shows the spectra of the 5 objects observed in 2006. The spectrum of B17 is very striking, with very strong He II $4686 \AA$ and $\mathrm{N}$ III emission, and a P-Cygni profile in $\mathrm{H} \beta$. All its lines are displaced by $>200 \mathrm{~km} \mathrm{~s}^{-1}$ with respect to other members and show an enormous shift in radial velocity with respect to the 2004 spectrum. We classify this object as an Ofpe star, almost certainly a binary, and will study it in detail in a future paper. A11 and A24 have He II $4542 \AA \simeq$ He I $4471 \AA$ and are therefore close to O7, while the in-filling of He II $4686 \AA$ indicates a moderate luminosity. A11 has N III 4630-4640 ̊ in emission, a wind feature typical of luminous stars. Based on the criteria laid out by Walborn \& Fitzpatrick (1990), we classify A11 as O7.5 Ib-II(f) and A24 as O6.5 III((f)). A38 has He II $4686 \AA$ strongly in absorption and we classify it as $08 \mathrm{~V}$, though it is close to $08.5 \mathrm{~V}$, if we use the quantitative criteria of Mathys (1988). Finally, A33 has weak He II $4686 \AA$ and $4542 \AA$, but no He II $4200 \AA$, and thus we classify it as B0.2 V. The accurate classifications agree quite well with the estimates obtained from the low-resolution spectra in the previous section.

Figure 3 shows the 3 classification spectra obtained in August 2007. The extremely prominent Si IV lines in A12 show it to be a luminous supergiant, while their ratio to Si III lines puts it at B0, in agreement with the presence of three weak He II lines. We adopt B0 Ia. A15 is similar to A11 and A24. He II $4686 \AA$ is more clearly in emission, but the lack of wind S IV emission lines and weak Si IV $4089 \AA$ prevent us from assigning a high luminosity. We settle for O7 Ibf. Finally, though clearly an O-type

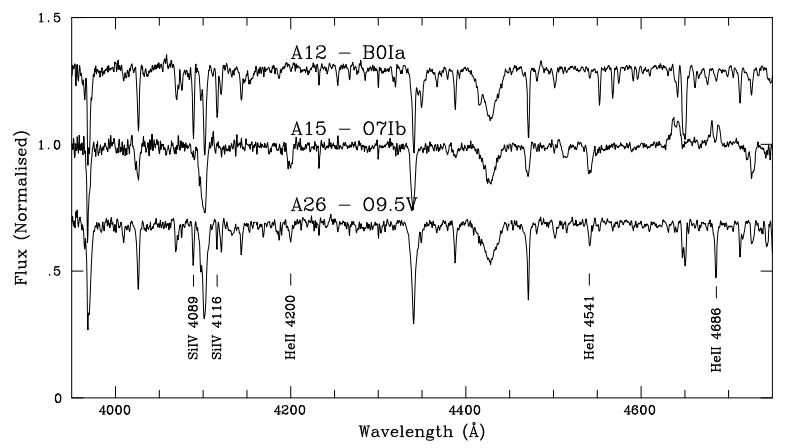

Fig. 3. Classification spectra of the 3 objects observed with the blue arm of ISIS on the WHT in August 2007. The most relevant lines are marked.

star because of the strong He II lines, A26 still shows many weak Si III and O II lines and is therefore O9.5 V.

We also obtained red spectra of 4 objects, covering the atmospheric window between $8300 \AA$ and $8900 \AA$, where spectral classification is possible (e.g., Clark et al. 2005). Figure 5 shows the spectra of three objects, B17 and two stars not observed in the blue, A18 and A25. The three spectra are similar and typical of late O-type stars. Only a few Paschen lines are visible, but the C III $8502 \AA$ line is clearly visible. This places the stars in the 07-09 range. The broadness of the Paschen lines and the small number visible indicates that A18 and A25 are not supergiants. We take an approximate spectral type $\sim$ O8. Based on their $K$ magnitudes and positions in the HR diagram (Sect. 4.3), A25 is likely a main-sequence object, but A18 could be a more evolved star.

A fourth object, B10 = MT 285, was also observed and its spectrum is displayed in Fig. 6. The strong asymmetric emission Paschen lines are typical of a Be star, with the very prominent O I $8446 \AA$ indicates that it is not a late-B object (Andrillat et al. 1988). The likely detection of He I $8779 \AA$ and strong O I $7774 \AA$ (not shown) emission indicates that it is B2 or earlier (Andrillat et al. 1988). This is fully consistent with the detection by Comerón et al. (2002) of He I $2.058 \mu \mathrm{m}$ in emission, as this is only seen in Be stars earlier than B3 (Clark \& Steele 2000). Therefore it is likely to be a massive Herbig Be star in Cyg OB2. None of the classical Be stars observed by Andrillat et al. (1988) shows $E W_{\mathrm{O}_{\mathrm{I}}}<-5.5 \AA$, but $\mathrm{B} 10$ has $E W_{\mathrm{O}_{\mathrm{I}}} \approx-10 \AA$ after correction for Pa 18, strongly suggesting that it is a Herbig Be object, as they tend to have stronger emission features.

\subsection{Model fits}

The WHT spectra, even if of moderate resolution, offer a good chance to complement the study presented by Herrero et al. (1999, 2002), expanding the sample that can be analysed. For all stars with blue WHT spectra except B17, which is unlikely to be a single star, we determined stellar parameters using FASTWIND (Santolaya-Rey et al. 1997; Puls et al. 2005), by fitting H and He line profiles in the standard way (Herrero et al. 1992; Repolust et al. 2004). The value of $v_{\infty}$ was adopted from the spectral type, after Kudritzki \& Puls (2000). We adopted a value of the microturbulence $\xi=10 \mathrm{~km} \mathrm{~s}^{-1}$ for all objects except A12 (the only B-supergiant in the sample) for which we adopted $15 \mathrm{~km} \mathrm{~s}^{-1} \cdot \beta$ (the exponent of the velocity law) was adopted to be 0.8 and varied when the fit could be improved. Again, only A12 needed a slightly larger value of $\beta$ (consistent with a slower wind acceleration), and we adopted $\beta=1.0$ for this source (but 
Table 2. Astrophysical parameters of programme stars, derived from model fits.

\begin{tabular}{lcccccccccc}
\hline \hline Name & Spectral type & $M_{V}$ & $\begin{array}{c}T_{\text {eff }} \\
(\mathrm{K})\end{array}$ & $\log g$ & $\begin{array}{c}R \\
\left(R_{\odot}\right)\end{array}$ & $\begin{array}{c}\dot{M} \\
\left(M_{\odot}\right)\end{array}$ & $\begin{array}{c}v_{\infty} \\
\left(\mathrm{km} \mathrm{s}^{-1}\right)\end{array}$ & $\begin{array}{c}v_{\text {rot }} \\
\left(\mathrm{km} \mathrm{s}^{-1}\right)\end{array}$ & $\begin{array}{c}\text { Mass } \\
\left(M_{\odot}\right)\end{array}$ & $\log \left(L / L_{\odot}\right)$ \\
\hline A11 & O7.5 Ib-II(f) & -5.8 & 36000 & 3.6 & 15.9 & $2.2 \times 10^{-6}$ & 1900 & $<160$ & 38.9 & 5.6 \\
A12 & B0 Ia & -6.7 & 27000 & 3.0 & 30.2 & $3.5 \times 10^{-6}$ & 1350 & 80 & 34.2 & 5.6 \\
A15 & O7 Ibf & -5.7 & 35000 & 3.2 & 15.6 & $3.2 \times 10^{-6}$ & 2100 & 245 & 19.0 & 5.5 \\
A24 & O6.5 III((f)) & -5.0 & 37500 & 3.6 & 10.7 & $1.7 \times 10^{-6}$ & 2600 & $<160$ & 18.1 & 5.3 \\
A26 & O9.5 V & -4.2 & 35000 & 3.9 & 7.7 & $4.1 \times 10^{-8}$ & 1300 & 90 & 17.6 & 4.9 \\
A33 & B0.2 V & -3.6 & 31000 & 4.0 & 6.6 & $2.0 \times 10^{-8}$ & 1000 & $<160$ & 16.6 & 4.6 \\
A38 & O8 V & -3.7 & 36000 & 4.0 & 6.0 & $4.9 \times 10^{-8}$ & 1900 & $<160$ & 13.8 & 4.7 \\
\hline
\end{tabular}

All models have been calculated using $\beta=0.8, v_{\text {turb }}=10 \mathrm{~km} \mathrm{~s}^{-1}$ and $\epsilon=0.09$, except for A12, which, being a B-type supergiant, required different wind parameters $\left(\beta=1.0, v_{\text {turb }}=15 \mathrm{~km} \mathrm{~s}^{-1}\right)$ and A15, which required $\epsilon=0.25$.

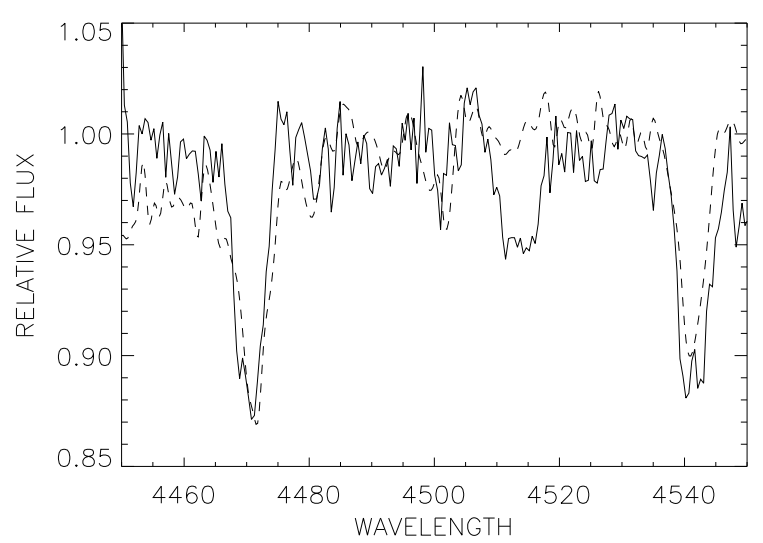

Fig. 4. The spectrum of A15 (O7 Ib; solid line) compared to that of A11 (O7.5 Ib-II(f), dotted line). The strong N III 4511-14 ̊̊ feature and the high value of $\epsilon$ derived from the model fits for A15 indicate advanced chemical evolution. The high rotational velocity and the underluminosity of this star all indicate anomalous evolution, perhaps due to mass transfer in a close binary.

note that lacking $\mathrm{H} \alpha$ or having low resolution, our data are not very sensitive to $\beta$ ). We should indicate that the final fit to the He II $4686 \AA$ line of A12 is not satisfactory.

Likewise, the He abundance by number relative to $\mathrm{H}$ plus $\mathrm{He}, \epsilon$, is set initially to the standard value $\epsilon=0.09$ for all stars, and varied to obtain better fits. Only A15 needed a higher value to fit the observed spectrum. The high value required by A15, $\epsilon=0.25$, points to an evolved object, consistently with its low gravity and its strong $\mathrm{N}$ spectrum. Figure 4 shows a comparison of the spectra of A11 and A15 (both O7 supergiants), around $4500 \AA$, where we can see the strong N III 4511-14 $\AA$ feature in the spectrum of A15. Note also that the projected rotational velocity is remarkably high $\left(245 \mathrm{~km} \mathrm{~s}^{-1}\right)$ for an evolved object (which is assumed to have lost significant amounts of angular momentum). The values derived suggest that the evolution of this object has been anomalous (perhaps as a consequence of binary evolution) as, in addition, it appears underluminous and undermassive for its spectral type.

The parameters derived are given in Table 2 and correspond very well to the spectral types derived in most cases. Errors in the stellar parameters are estimated at $\delta T_{\text {eff }}= \pm 1500 \mathrm{~K}, \delta \log g=$ \pm 0.2 and $\delta(\log \dot{M})= \pm 0.3$ for the low resolution observations and slightly lower in $T_{\text {eff }}( \pm 1000 \mathrm{~K})$ and $\log g( \pm 0.15)$ for the higher resolution data. Absolute luminosities, radii and masses have been calculated from the $K_{\mathrm{S}}$ magnitude, assuming $D M=$ $10.8(d=1.4 \mathrm{kpc})$, after Hanson (2003), following the method discussed in Sect. 4.3. In the case of A38, the value $M_{V}=-3.7$ is

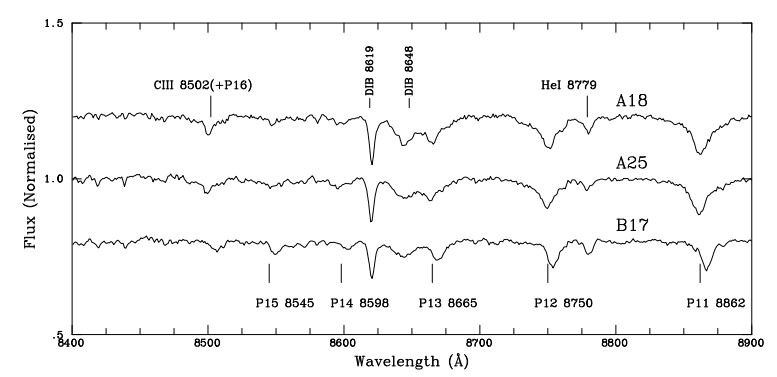

Fig. 5. I-band spectra of three targets observed with the WHT in August 2007. A25 does not show clear evidence for any Paschen line beyond Pa 13 and has weak C III $8502 \AA$. It is hence most likely an O8-9 V star. A18 is clearly more luminous and probably earlier. Note the important radial velocity shifts in the lines of B17, this time in the opposite sense to those in the blue spectrum in Fig. 2.

more than half a magnitude fainter than expected for the spectral type, resulting in the low derived mass and luminosity.

Note that, in order to compare the absolute astrophysical parameters derived for these objects to those in previous works (Herrero et al. 1999, 2002), they must be reduced to the same distance, as previous works assumed the canonical $D M=11.2$.

Projected rotational velocities could not be determined for the stars observed in 2006 due to the low resolution; the instrumental profile dominates the line broadening for the metals ( $\mathrm{H}$ and $\mathrm{He}$ lines are broadened by the Stark profile). However, this provides an upper limit, as instrumental broadening dominates in all our objects, allowing us to ascertain that they all rotate with $v \sin i<160 \mathrm{~km} \mathrm{~s}^{-1}$.

\section{Discussion}

\subsection{Completeness}

Our results confirm the enormous success of Comerón et al. (2002) at identifying reddened OB stars. Only one of the candidates turns out to be an interloper. The important point, however, is estimating whether these objects are members of Cyg OB2. The line of sight in this direction runs parallel to the Local Arm, and populations at different distances may lie projected together. While it is extremely unlikely that early O-type might be found far away from massive clusters or associations, except for a few runways (cf. de Wit et al. 2005; van den Bergh 2004), less massive stars will certainly be found if one looks through a Galactic Arm.

In this sense, our sample appears rather different from that of Hanson (2003), who observed only candidates which were bright in $B$, most of which turned out to be foreground B-type 


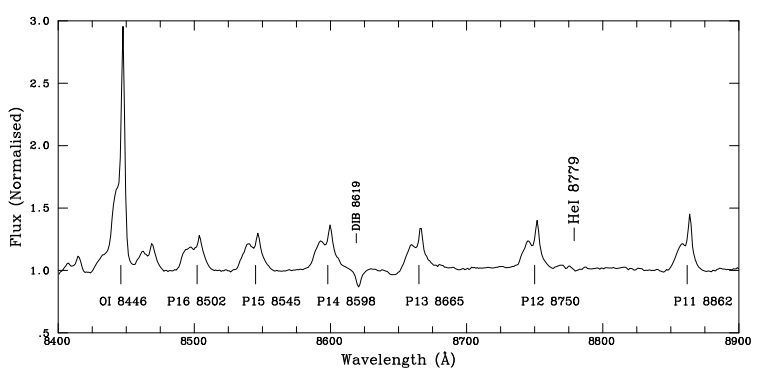

Fig. 6. $I$-band spectrum of B10. The data available identify it as an early $(\leq \mathrm{B} 2)$ Be star, but do not allow us to decide whether it is a classical Be star or a PMS Herbig Be object. The enormous strength of O I $8446 \AA$, though, is unusual for a classical Be star and points to the second option.

stars. Even though this is not surprising for the August 2007 sample, which was selected on the basis of bright $K$ magnitudes, it is more striking for the first (Loiano) sample, which simply consists of objects somewhat fainter in $B$ than those observed by Hanson (2003). This suggests that, even though extinction is clearly variable across the face of Cyg OB2, on average, there is a range of extinctions where we can find members, and this translates into a range of magnitudes (according to spectral type). This range has been estimated as $4 \lessgtr A_{\mathrm{V}} \lesssim 7$ by previous authors (e.g., Massey \& Thompson 1991), and may extend to somewhat higher values when stars from the list of Comerón et al. (2002) are added.

Because of this, we suggest that there cannot be many more O-type members amongst the candidates given by Comerón et al. (2002), though there must be some (for example, perhaps A17, see below). Very few of them are likely to be intrinsically bright (and so very massive, evolved) members of the cluster. Based on their $\left(J-K_{\mathrm{S}}\right)$ colours and $K_{\mathrm{S}}$ magnitudes, only A4 and A8 might be sufficiently bright (intrinsically) to be obscured O-type giants or supergiants.

In addition, our data reveal three new evolved O-type stars (A11, A15 and A24), which help define the main-sequence turnoff of the association. A11 is of particular interest, as it lies very close to the Blue Hypergiant (BHG) candidate \#12, in what likely is the most obscured part of the association. We classify it $07.5 \mathrm{Ib}-\mathrm{II}(\mathrm{f})$, as it almost looks evolved enough to be a supergiant, and its analysis indeed shows that it is a very massive star. Comerón et al. (2002) suggest it may be the counterpart to the X-ray source 1E 2023043+4103.9. Another candidate from Comerón et al. (2002), A17, lies very close to it. It has very similar IR colours, but is two magnitudes fainter in $K$. This is most likely a late-O/early-B main-sequence member.

\subsection{Clustering}

Figure 8 shows a 2 MASS $K_{\mathrm{S}}$ image of the central region of Cyg OB2, containing the two cluster-like groupings identified by Bica et al. (2003) and the area around \#12. The two clusters are prominent against the background. The field shown in Fig. 8 is $\sim 12^{\prime} \times 12^{\prime}$, corresponding to $\sim 5 \mathrm{pc}$ at $1.4 \mathrm{kpc}$. The separation between Cluster 1 and Cluster $2\left(\lessgtr 6^{\prime}\right)$ is equivalent to $2.5 \mathrm{pc}$, and so smaller than the radius of relatively massive clusters in the Perseus Arm, such as h Per or NGC 663.

The nine brightest stars in Cluster 2 have $(J-K)_{\mathrm{S}}=0.63 \pm$ 0.03 (standard deviation) and eight of them have $(J-K)_{\mathrm{S}}$ between 0.59 and 0.63 . This uniformity in reddening represents strong confirmation of their association, also clear in the colour-magnitude diagram shown by Bica et al. (2003). Cluster 1 , on the other hand, does not seem to have a uniform reddening, but we do not think that this is a strong argument against its reality, in this region of patchy obscuration.

In addition to these two groups, and outside the field covered by Fig. 8, there is another obvious region of stellar overdensity present in the data of Kiminki et al. (2007) - surrounding star \#4 (O7 III). It comprises MT213 (B0 V), MT215 (B2 V), MT216 (B1.5 V) and MT221 (B2 V). MT187 (B1 V), MT227 (O9V), MT241 (B2 V) and MT258 (O8 V) also lie within $3^{\prime}$. MT187 and MT221 are significantly ( $>0.1 \mathrm{mag}$ ) more reddened than the others, but the other seven have $(J-K)_{\mathrm{S}}=0.49 \pm 0.03$, again strongly hinting at a real physical association.

The presence of all these small groups over a large area suggests that star formation has proceeded in small bouts in this region, perhaps over an extended period of time. In spite of this, the bulk of the population occupies positions in the HR diagram incompatible with a very long period of star formation. Subclustering is seen in the largest Galactic star forming regions, such as W49A (Homeier \& Alves 2005) or W51 (Nanda-Kumar et al. 2004). Study of large stellar complexes in M51 (Bastian et al. 2005) shows that the age spread within different clusters is $\lesssim 10$ Myr.

\subsection{HR diagram and ages}

As discussed by Hanson (2003), the main sequence in Cyg OB2 extends clearly down to O6 V. Star \#22, classified O4 III(f) by Massey \& Thompson (1991) has been shown to be a close double containing an $\mathrm{O} 3$ If* supergiant and an O6 V star (Walborn et al. 2002). MT516, classified as O5.5 V((f)) by Massey \& Thompson (1991), was found to have a rather low gravity by Herrero et al. (1999). Indeed, the fact that He II $4542 \AA$ is somewhat stronger than He II $4686 \AA$ shows that this object is rather far away from the ZAMS. Therefore this star is likely better classified as O5.5 III, joining \#8C and the faint component of \#8A as an object still on the main sequence, but already showing some signs of evolution. Therefore the age of the association would seem to be set by the fact that stars more massive than $\mathrm{O} 6 \mathrm{~V}$ are already somewhat evolved, while O6 V stars are not.

However, within a classical theory of stellar evolution, it is difficult to see how this fits with the presence of $\mathrm{O} 3$ supergiants. In order to address this question and also exploit the potential of Cyg OB2 as a laboratory, we have constructed an HR diagram utilising the wealth of new spectral type determinations in this region. We have used the 2MASS $J H K_{\mathrm{S}}$ magnitudes for all objects and their spectral types (from Kiminki et al. 2007; Hanson 2003, or this work) in order to place them in a semiobservational HR diagram. We have followed the procedure used by, for instance, Massey et al. (1995), but taking infrared rather than optical magnitudes. We have resorted to $J H K_{\mathrm{S}}$ magnitudes partly because many stars of interest lack good $U$-band photometry, but also because this allows a test of the usefulness of infrared data to study obscured massive clusters.

From the spectral types derived, we have taken a $T_{\text {eff }}$ and bolometric correction $B C$, using the calibration of Martins et al. (2005) for O-type stars and that of Humphreys \& McElroy (1984) for B-type stars (the two calibrations agree quite well around $\mathrm{B} 0$; however, the possible existence of an artificial jump between $\mathrm{B} 0$ and $\mathrm{B} 1$ has been noted by previous authors). We also take intrinsic $(V-K)_{0}$ and $(J-K)_{0}$ colours from the calibration of Wegner (1994). With the observed $\left(J-K_{\mathrm{S}}\right)$, we derive $E\left(J-K_{\mathrm{S}}\right)$. As the reddening to the association is known to 


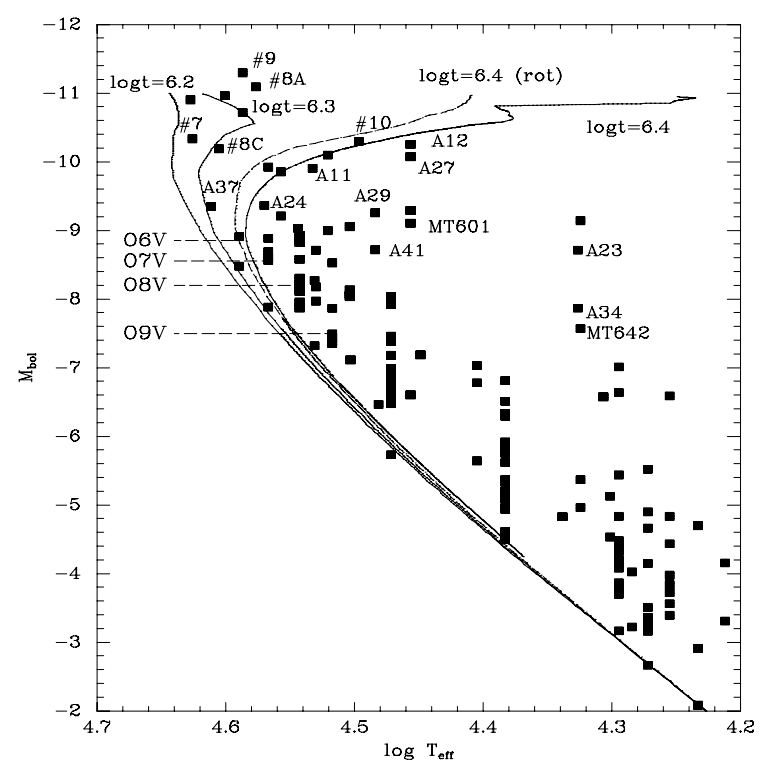

Fig. 7. Semi-observational HR diagram for Cyg OB2, based on published spectral types and 2MASS $J H K_{\mathrm{S}}$ photometry (see text for details). Continuous lines are non-rotating isochrones for $\log t=6.2$, 6.3 and 6.4 from Schaller et al. (1992). The dashed line is the $\log t=$ 6.4 isochrone in the high-rotation models (Meynet \& Maeder 2003).

be very close to standard (Hanson 2003), we simply calculate $A_{K_{\mathrm{S}}}=0.67 E\left(J-K_{\mathrm{S}}\right)$.

We then calculate $K_{0}=K_{\mathrm{S}}-A_{K_{\mathrm{S}}}-D M$, using $D M=10.8$ from Hanson (2003), and by adding $(V-K)_{0}$ and the $B C$, arrive at a semi-observational $M_{\mathrm{Bol}}$. Figure 7 plots $M_{\mathrm{Bol}}$ against the $T_{\mathrm{eff}}$ derived from the spectral classification. Superposed on it, are Geneva isochrones without rotation for $\log t=6.2$ (1.5 Myr), $\log t=6.3(2 \mathrm{Myr})$ and $\log t=6.4(2.5 \mathrm{Myr})$, as well as the rotating isochrone for $\log t=6.4$. We tried to fit the data using the higher distance modulus $(D M=11.3)$ obtained by averaging spectroscopic distances (Kiminki et al. 2007), but this left all the stars well above the ZAMS. The data used for this diagram are listed in Table 3.

The diagram shows several notable features. There is a very well traced main sequence extending to the $\mathrm{O} 6 \mathrm{~V}$ stars. As noted by Hanson (2003), A37 (O5 V) is earlier than any other MS stars, but seems to fit the sequence well. Around $\log T_{\text {eff }} \sim 4.3$, there lie a number of B stars well above the main sequence, unlikely to be connected with the rest of the population. Objects like MT642 (B1 III), A23 (B0.7 Ib) or A34 (B0.7 Ib) are very probably not members of Cyg OB2.

There are two evolutionary sequences that seem to turn off the main sequence. The one below the isochrones is formed by MT138 (O8 I), A32 (O9.5 IV), A41 (O9.7 II), A29 (O9.7 Iab), A36 (B0 Ib), and MT601 (B0 Iab). Though this sequence may provide a decent fit to the $\log t=6.7$ (5 Myr) isochrone, the random distribution in luminosity class suggests that this is not a real evolutionary sequence, but simply the projection of a number of luminous stars situated at slightly different distances. It is worthwhile mentioning, though, that many of these objects have distance moduli comparable to that of the main Cyg OB2 association.

In contrast, the sizable population of evolved stars lying around the $\log t=6.4$ isochrone seems to form a much more coherent group. Moving along the isochrone, we have A24 (O6.5 III) and \#4 (O7 III), \#8B (O7 II-III), A11 (O7.5 Ib-II), A20 (O8 II), \#10 (=MT632, O9.5 Ia), A12 (B0 Ia) and A27 (B0 Ia).
The excellent progression in luminosity class with spectral type strongly supports the hypothesis that these objects are really following the isochrone. Only two stars with accurate spectral types do not fit this evolutionary sequence: one is MT771 (O7 V), which appears as bright as \#4. This is easily explained by the fact that it is a double-lined spectroscopic binary with two similar components (Kiminki et al. 2007). The other one is A15 (O7 Ib), which is $\sim 0.6$ mag fainter than expected. As mentioned, the analysis of its spectrum reveals very high $\mathrm{He}$ and $\mathrm{N}$ abundances, and a very low mass for its spectral type. Therefore this is indeed a peculiar object, perhaps the product of mass transfer in a close binary, as \#5 and B17 are also likely to be.

The distribution of main sequence stars, the main-sequence turn-off and the sequence of evolved stars strongly supports an age $2.5 \mathrm{Myr}$ for the bulk of Cyg OB2. According to the calibration of Martins et al. (2005), this implies that stars up to $\sim 35 M_{\odot}$ are still close to the ZAMS, while more massive stars are already more evolved. However, it is obvious that the brightest stars in the association fall well above the adopted isochrone.

Given the extent of Cyg OB2, the possibility of a spread in ages cannot be excluded and may even seem logical. Indeed, in a recent paper, Drew et al. (2008) have shown an important concentration of A-type stars to the South of the bulk of the O-type stars. In order to be at the same distance as the O-star association, these A-type stars must be part of a 5-7 Myr population.

Does the presence of $\mathrm{O} 3$ supergiants indicate the existence of an even younger population? Certainly this possibility cannot be excluded, but it is worth taking in consideration two points:

- if there is an age difference, we would expect to find some sort of spatial segregation between the older and younger population, but this is not evident in the data. The area shown in Fig. 8 contains most of the earliest objects, but also A11 (O7.5 Ib-II) and \#12. The moderately evolved \#8B (O7 II-III) falls just in the middle of Cluster 2, which contains three of the early objects;

- stars more massive than the O6 V objects still in the main sequence appear as either earlier-type supergiants (Of* stars) or intermediate luminosity O6-7 stars. In other words, if there is a younger population, all its members appear as Of* stars just now.

At the estimated age, it is unlikely that any stars might have undergone supernova, and indeed no supernova remnant is seen in the area (Pasquali et al. 2002). All the stars in Fig. 7 occupy positions in the HR diagram compatible with being still in the hydrogen core-burning phase. The BHG candidate \#12 may be past this phase and there are 5 Wolf-Rayet stars in the region that have been proposed as possible members (Pasquali et al. 2002). The WC stars WR 144 and WR 146 may actually be the descendants of the most massive stars in the association (Pasquali et al. 2002). The fact that some of the most massive stars appear as O3 If supergiants, while others are moving towards the red part of the HR diagram (or seem already to be locked in an LBV phase, like \#12) is highly suggestive of the idea that not all very massive stars evolve in the same way.

There is, however, ample evidence suggesting that star formation has been going on for quite some time in a large area around the recognizable core of Cygnus OB2. Indeed many of the evolved massive stars that are unlikely to belong to the current generation of massive stars lie at approximately the same distance and could belong to an older ( $7 \mathrm{Myr})$ generation, associated with the young A-type stars detected by Drew et al. (2008). In the massive association 30 Dor, Walborn \& Blades (1997) also find a population of OB stars somewhat older 
(4-6 Myr) than those in the main cluster R136 scattered across the entire complex. Likewise, Mokiem et al. (2007) find ages of $7.0 \pm 1.0$ and $3.0 \pm 1.0 \mathrm{Myr}$ for the associations LH9 and LH10, in the giant H II region N11 in the LMC. Their data are consistent with LH9 having triggered star formation in LH10.

\section{Conclusions}

Though the candidate sample of Comerón et al. (2002) contains a high fraction of likely non-members, as discussed by Hanson (2003), it has also allowed the detection of a number of obscured $\mathrm{O}$ stars and very luminous B0 Ia supergiants very likely to be members of Cyg OB2.

When these objects are included in the HR diagram, it becomes clear that there is a sequence of moderately evolved stars detaching from the main sequence exactly at the position where we stop seeing luminosity class $\mathrm{V}$ objects, i.e., around $\mathrm{O6} \mathrm{V}$. These two facts combined support an age of $\sim 2.5 \mathrm{Myr}$ for the bulk of the association.

The HR diagram presented in Fig. 7 contains the largest number of Cyg OB2 members ever displayed in such a diagram. It contains $\sim 50$ stars that may have started their lives as mainsequence O-type stars and only a few of these are unlikely to be members. Unless a population of extremely obscured O-type stars is lying at fainter magnitudes than probed by 2MASS, the total number of O-type stars in the association is almost certain to be in the $60-70$ range.

The number of stars that have already left the main sequence and lie above the $\mathrm{O} 6 \mathrm{~V}$ members that define the turn-off is more securely determined. If the main association is basically co-eval, these represent the subset of stars that were originally more massive than $35 M_{\odot}$. Counting \#12, which is not shown in Fig. 7 because of its claimed spectral variability (Kiminki et al. 2007), there are 21 such stars. The evolved interacting binaries \#5 and B17 (not in Fig. 7) should be counted too (perhaps doubly). The resulting number is certainly only a lower limit. Apart from possible unrecognised close doubles and binaries, at least two of the Wolf-Rayet stars in the area are likely to be descendants of very massive stars (Pasquali et al. 2002). Also, Comerón \& Pasquali (2007) identify BD $+53^{\circ} 3654$ as a likely runaway O4 If member of the association. Therefore, we have identified a population of at least 25 stars that were originally more massive than $35 M_{\odot}$. Given the uncertainties - in particular the very high binary fraction (Kiminki et al. 2007) - we refrain from trying to derive a total mass for the association by assuming an IMF.

The brightest members, with spectral types in the O3-O5 range, may technically be considered blue stragglers. Though a real age difference cannot be ruled out, it does not seem to be borne out by the spatial distribution of stars, perhaps suggesting that we are seeing stars of similar mass evolve in very different ways.

Three luminous supergiants (\#10 O9.5 Ia, A12 B0 Ia and A27 B0 Ia) seem to follow the $2.5 \mathrm{Myr}$ isochrone and so appear to be the descendants of stars more massive than $\sim 40 \mathrm{M}_{\odot}$. This is in agreement with an initial mass estimate of $48 M_{\odot}$ for \#10 (Herrero et al. 2002), which may have to be slightly reduced if the lower $D M=10.8$ is adopted. These objects will probably soon reach the LBV instability, which \#12 is perhaps already encountering. A large population of O9-B1 Ia supergiants descended from stars with $M_{*} \approx 35 M_{\odot}$ is found in the older ( 4.5 Myr) cluster Westerlund 1 together with a number of LBVs and Yellow Hypergiants (Clark et al. 2005).

In summary, even if Cyg OB2 falls short of the proposed 100 O-type stars by a factor of $\sim 2$, its nuclear region still represents one of the most massive collections of early-type stars known in the Galaxy and its relatively low reddening cements its value as a laboratory for the study of their properties.

Acknowledgements. We thank Vanessa Stroud for help with the 2007 run and reduction of some spectra. During most of this work, IN was a researcher of the programme Ramón y Cajal, funded by the Spanish Ministerio de Educación y Ciencia and the University of Alicante, with partial support from the Generalitat Valenciana and the European Regional Development Fund (ERDF/FEDER). This research is partially supported by the MEC under grants AYA2005-00095, AYA2004-08271-C02-01, AYA2007-67456-C02-01 and CSD2006-70 and by the Generalitat Valenciana under grant GV04B/729. The Cassini telescope is operated at the Loiano Observatory by the Osservatorio Astronomico di Bologna. The WHT is operated on the island of La Palma by the Isaac Newton Group in the Spanish Observatorio del Roque de Los Muchachos of the Instituto de Astrofísica de Canarias. The June 2006 observations were taken as part of the service programme (programme SW2005A20). This research has made use of the Simbad data base, operated at CDS, Strasbourg (France). This publication makes use of data products from the Two Micron All Sky Survey, which is a joint project of the University of Massachusetts and the Infrared Processing and Analysis Center/California Institute of Technology, funded by the National Aeronautics and Space Administration and the National Science Foundation.

\section{References}

Andrillat, Y., Jaschek, M., \& Jaschek, C. 1988, A\&AS, 72, 129

Bastian, N., Gieles, M., Efremov, Yu. N., \& Lamers, H. J. G. L. M. 2005, A\&A, 443, 79

van den Bergh, S. 2004, AJ, 128, 1880

Bica, E., Bonatto, Ch., \& Dutra, C. M. 2003, A\&A, 405, 991

Clark, J. S., \& Steele, I. A. 2000, A\&AS, 141, 65

Clark, J. S., Negueruela, I., Crowther, P. A., \& Goodwin, S. P. 2005, A\&A, 434, 949

Comerón, F., \& Pasquali, A. 2005, A\&A, 430, 541

Comerón, F., \& Pasquali, A. 2007, A\&A, 467, L23

Comerón, F., Pasquali, A., Rodighiero, G., et al. 2002, A\&A, 389, 874

Draper, P.W., Taylor, M., \& Allan, A. 2000, Starlink User Note 139.12, R.A.L.

Drew, J. E., Greimel, R., Irwin, M. J., \& Sale, S. E. 2008, MNRAS, 386, 1761

Ducati, J. R., Bevilacqua, C. M., Rembold, S. B., \& Ribeiro, D. 2001, ApJ, 558, 309

Figer, D. F., Najarro, F., Gilmore, D., et al. 2002, ApJ, 581, 258

Fitzpatrick, E. L. 1999, PASP, 111, 63

Hanson, M. M. 2003, ApJ, 597, 957

Herrero, A., Kudritzki, R. P., Vilchez, J. M., et al. 1992, A\&A, 261, 209

Herrero, A., Corral, L. J., Villamariz, M. R., \& Martín, E. L. 1999, A\&A, 348, 542

Herrero, A., Puls, J., \& Najarro, F. 2002, A\&A, 396, 949

Homeier, N. L., \& Alves, J. 2005, A\&A, 430, 481

Howarth, I., Murray, J., Mills, D., \& Berry, D. S. 1998, Starlink User Note 50.21, R.A.L.

Humphreys, R. M., \& McElroy, D. B. 1984, ApJ, 284, 565

Johnson, H. L., \& Morgan, W. W. 1954, ApJ, 119, 344

Kiminki, D. C., Kobulnicky, H. A., Kinemuchi, K., et al. 2007, ApJ, 664, 1102 Knödlseder, J. 2000, A\&A, 360, 539

Koornneef, J. 1985, A\&A, 128, 84

Kudritzki, R. P., \& Puls, J. 2000, ARA\&A, 38, 613

Martins, F., Schaerer, D., \& Hillier, J. 2005, A\&A, 436, 1049

Massey, P., \& Thompson, A. B. 1991, AJ, 101, 1408

Massey, P., Johnson, K. E., \& DeGioia-Eastwood, K. 1995, ApJ, 454, 151

Mathys, G. 1988, A\&AS, 76, 427

Meynet, G., \& Maeder, A. 2003, A\&A, 404, 975

Mokiem, M. R., de Koter, A., Evans, C. J., et al. 2007, A\&A, 465, 1003

Nanda-Kumar, M. S., Kamath, U. S., \& Davis, C. J. 2004, MNRAS, 353, 1025

Pasquali, A., Comerón, F., Gredel, R., et al. 2002, A\&A, 396, 533

Puls, J., Urbaneja, M. A., Venero, R., et al. 2005, A\&A, 435, 669

Repolust, T., Puls, J., \& Herrero, A. 2004, A\&A, 415, 349

Rieke, G. H., \& Lebofsky, M. J. 1985, ApJ, 288, 618

Santolaya-Rey, A. E., Puls, J., \& Herrero, A. 1997, A\&A, 323, 488

Schaller, G., Schaerer, D., Meynet, G., \& Maeder, A. 1992, A\&AS, 96, 269

Shortridge, K., Meyerdicks, H., Currie, M., et al. 1997, Starlink User Note 86.15, R.A.L.

Skrutskie, M. F., Cutri, R. M., \& Stiening, R. 2006, AJ, 131, 1163

Walborn, N. R., \& Blades, J. C. 1997, ApJS, 112, 457

Walborn, N. R., \& Fitzpatrick, E. L. 1990, PASP, 102, 379

Walborn, N. R., Howarth, I. D., Lennon, D. J., et al. 2002, AJ, 123, 2754

Wegner, W. 1994, MNRAS, 270, 229

de Wit, W. J., Testi, L., Palla, F., \& Zinnecker, H. 2005, A\&A, 437, 247 
I. Negueruela et al.: New very massive stars in Cygnus OB2, Online Material p 1

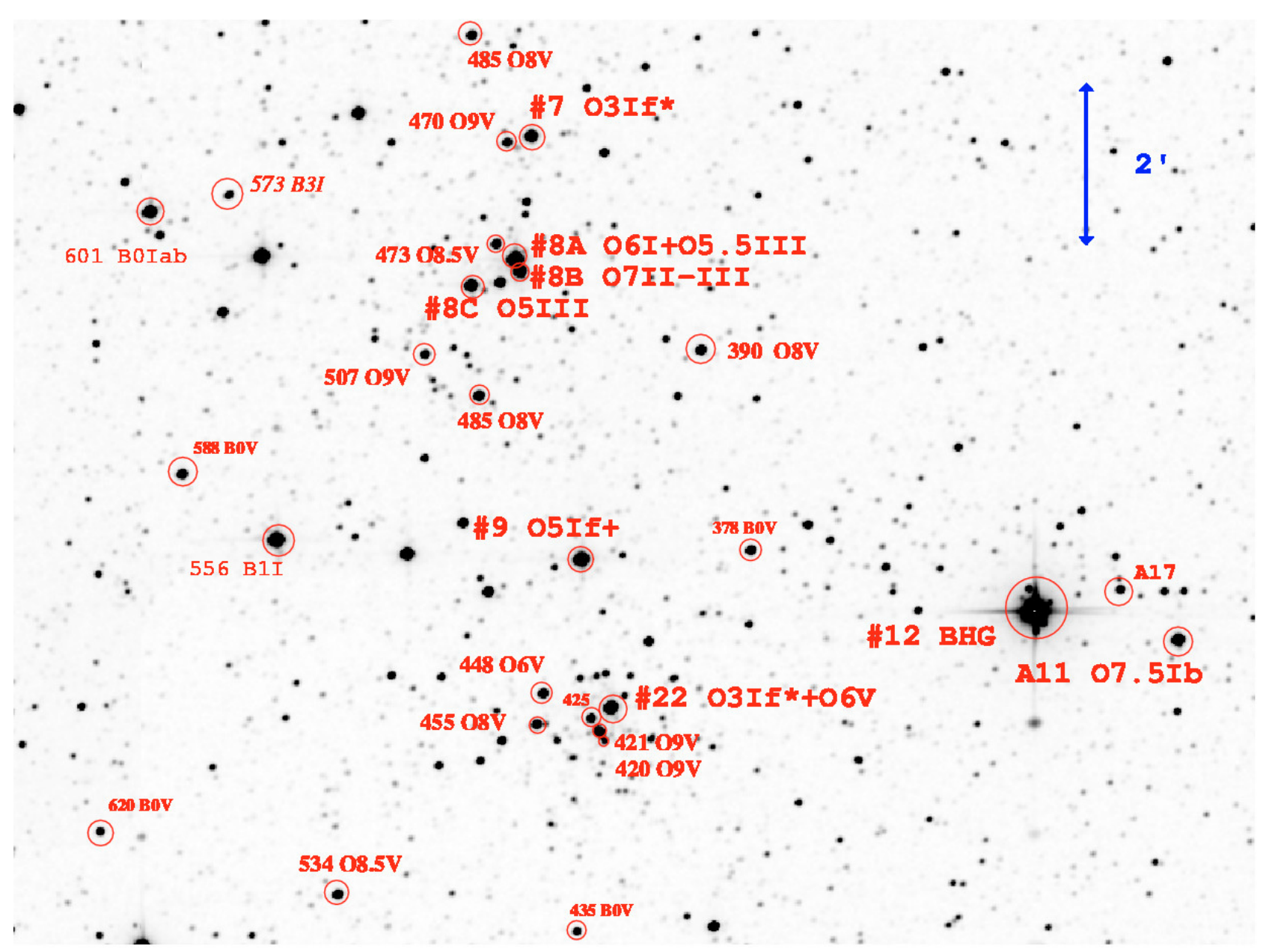

Fig. 8. A 2 MASS $K_{\mathrm{S}}$ image of the central region of Cyg OB2. All catalogued stars more massive than B0 V are marked. MT573 (B3 I) is obviously a background object, while the connection of MT556 (B1 I) and MT601 (B0 Iab) to the association is unclear. They may represent an older ( $~ 8 \mathrm{Myr}$ ) population at about the same distance. The two cluster-like groupings identified by Bica et al. (2003) stand out in the image. Object 1 is centred on MT425 and contains \#22, while Object 2 is centred on the three components of \#8. 
I. Negueruela et al.: New very massive stars in Cygnus OB2, Online Material p 2

Table 3. Adopted data for stars in Fig. 7. Spectral types are from Kiminki et al. (2007), Hanson (2003) or here.

\begin{tabular}{|c|c|c|c|c|c|c|c|c|c|c|}
\hline Name & $\begin{array}{l}\text { Spectral } \\
\text { type }\end{array}$ & $\log T_{\text {eff }}$ & B.C. & $\bar{J}$ & $\overline{K_{\mathrm{s}}}$ & $E(J-K)$ & $\overline{M_{K}}$ & $\overline{K_{0}}$ & $\overline{V_{0}}$ & $M_{\text {bol }}$ \\
\hline$\# 1$ & O8 V & 4.54 & -4.14 & 7.97 & 7.37 & 0.76 & 6.85 & -3.95 & -4.79 & -8.92 \\
\hline \#2 & B1 I & 4.31 & -2.51 & 8.08 & 7.63 & 0.58 & 7.24 & -3.56 & -4.07 & -6.57 \\
\hline MT70 & $09 \mathrm{~V}$ & 4.52 & -3.99 & 8.61 & 7.75 & 1.04 & 7.05 & -3.75 & -4.54 & -8.53 \\
\hline MT145 & 09 III & 4.50 & -3.76 & 9.07 & 8.63 & 0.62 & 8.22 & -2.58 & -3.35 & -7.11 \\
\hline \#4 & O7 IIIf & 4.56 & -4.22 & 7.58 & 7.11 & 0.67 & 6.66 & -4.14 & -4.99 & -9.21 \\
\hline MT227 & O9 V & 4.52 & -3.99 & 8.71 & 8.19 & 0.71 & 7.71 & -3.09 & -3.88 & -7.87 \\
\hline MT220 & B1 V & 4.38 & -3.13 & 10.94 & 10.23 & 0.86 & 9.65 & -1.15 & -1.81 & -4.93 \\
\hline MT202 & B2 V & 4.29 & -2.55 & 11.18 & 10.57 & 0.73 & 10.08 & -0.72 & -1.31 & -3.86 \\
\hline MT103 & B1 V & 4.38 & -3.13 & 9.74 & 8.94 & 0.96 & 8.30 & -2.50 & -3.16 & -6.29 \\
\hline MT221 & B2 V & 4.29 & -2.55 & 10.64 & 10.04 & 0.71 & 9.56 & -1.24 & -1.83 & -4.38 \\
\hline MT216 & $\mathrm{B} 1.5 \mathrm{~V}$ & 4.34 & -2.86 & 10.35 & 9.86 & 0.61 & 9.45 & -1.35 & -1.97 & -4.83 \\
\hline MT215 & B2 V & 4.29 & -2.55 & 10.65 & 10.19 & 0.58 & 9.80 & -1.00 & -1.59 & -4.14 \\
\hline MT213 & B0 V & 4.47 & -3.78 & 9.52 & 9.07 & 0.63 & 8.65 & -2.15 & -2.91 & -6.69 \\
\hline MT191 & B3 IV & 4.23 & -2.02 & 11.74 & 9.91 & 1.93 & 8.61 & -2.19 & -2.68 & -4.70 \\
\hline MT129 & B3 V & 4.27 & -2.39 & 11.32 & 10.67 & 0.75 & 10.17 & -0.63 & -1.12 & -3.51 \\
\hline MT108 & B3 IV & 4.23 & -2.02 & 12.32 & 11.71 & 0.71 & 11.23 & 0.43 & -0.06 & -2.08 \\
\hline MT174 & B2 IV & 4.26 & -2.21 & 9.80 & 9.23 & 0.68 & 8.77 & -2.03 & -2.62 & -4.83 \\
\hline MT186 & B2 Ve & 4.29 & -2.55 & 11.21 & 10.63 & 0.69 & 10.17 & -0.63 & -1.22 & -3.77 \\
\hline MT169 & B2 V & 4.29 & -2.55 & 11.12 & 10.61 & 0.62 & 10.20 & -0.60 & -1.19 & -3.75 \\
\hline MT5 & O6 V & 4.59 & -4.47 & 9.10 & 8.31 & 0.95 & 7.68 & -3.12 & -4.01 & -8.48 \\
\hline MT42 & B2 V & 4.29 & -2.55 & 10.86 & 10.24 & 0.73 & 9.75 & -1.05 & -1.64 & -4.20 \\
\hline MT21 & B2 II & 4.26 & -2.20 & 10.86 & 10.30 & 0.66 & 9.86 & -0.94 & -1.36 & -3.56 \\
\hline MT20 & B0 V & 4.47 & -3.78 & 9.79 & 8.89 & 1.09 & 8.16 & -2.64 & -3.40 & -7.18 \\
\hline MT97 & B2 V & 4.29 & -2.55 & 11.81 & 11.24 & 0.68 & 10.78 & -0.02 & -0.61 & -3.16 \\
\hline МТ179 & B3 V & 4.27 & -2.39 & 10.67 & 10.03 & 0.74 & 9.53 & -1.27 & -1.76 & -4.15 \\
\hline MT164 & B3 V & 4.27 & -2.39 & 12.07 & 11.48 & 0.69 & 11.01 & 0.21 & -0.28 & -2.66 \\
\hline MT106 & B3 V & 4.27 & -2.39 & 11.62 & 11.00 & 0.72 & 10.52 & -0.28 & -0.77 & -3.16 \\
\hline MT138 & O8 I & 4.52 & -3.99 & 8.07 & 7.26 & 0.99 & 6.60 & -4.20 & -5.01 & -9.00 \\
\hline MT200 & B3 V & 4.27 & -2.39 & 9.62 & 8.79 & 0.94 & 8.16 & -2.64 & -3.13 & -5.52 \\
\hline MT222 & B3 V & 4.27 & -2.39 & 11.50 & 10.83 & 0.77 & 10.32 & -0.48 & -0.97 & -3.36 \\
\hline MT311 & B2 V & 4.29 & -2.55 & 10.68 & 10.00 & 0.78 & 9.48 & -1.32 & -1.91 & -4.46 \\
\hline MT252 & B1.5 III & 4.30 & -2.53 & 10.66 & 9.97 & 0.86 & 9.40 & -1.40 & -2.00 & -4.53 \\
\hline MT322 & B2.5 V & 4.28 & -2.39 & 11.82 & 11.08 & 0.85 & 10.51 & -0.29 & -0.83 & -3.22 \\
\hline MT292 & B2 V & 4.29 & -2.55 & 9.73 & 9.04 & 0.80 & 8.51 & -2.29 & -2.88 & -5.44 \\
\hline MT255 & B2 III & 4.26 & -2.20 & 11.47 & 10.76 & 0.89 & 10.17 & -0.63 & -1.19 & -3.39 \\
\hline MT248 & B2 V & 4.29 & -2.55 & 10.47 & 9.92 & 0.66 & 9.48 & -1.32 & -1.91 & -4.46 \\
\hline MT234 & B2 V & 4.29 & -2.55 & 10.45 & 9.90 & 0.65 & 9.46 & -1.34 & -1.93 & -4.48 \\
\hline MT238 & B1 V & 4.38 & -3.13 & 11.39 & 10.67 & 0.87 & 10.09 & -0.71 & -1.37 & -4.50 \\
\hline MT298 & B3 V & 4.27 & -2.39 & 11.54 & 10.92 & 0.73 & 10.43 & -0.37 & -0.86 & -3.25 \\
\hline MT250 & B2 III & 4.26 & -2.20 & 10.43 & 9.99 & 0.61 & 9.58 & -1.22 & -1.78 & -3.97 \\
\hline MT259 & B0 Ib & 4.46 & -3.60 & 9.19 & 8.77 & 0.57 & 8.39 & -2.41 & -3.00 & -6.60 \\
\hline MT268 & B2.5 V & 4.28 & -2.39 & 10.94 & 10.24 & 0.81 & 9.70 & -1.10 & -1.64 & -4.02 \\
\hline MT241 & B2 V & 4.29 & -2.55 & 10.75 & 10.27 & 0.60 & 9.87 & -0.93 & -1.52 & -4.08 \\
\hline MT258 & O8 V & 4.54 & -4.14 & 8.54 & 8.02 & 0.67 & 7.57 & -3.23 & -4.07 & -8.21 \\
\hline MT295 & B2 V & 4.29 & -2.55 & 11.12 & 10.56 & 0.67 & 10.12 & -0.68 & -1.27 & -3.83 \\
\hline \#6 & $08 \mathrm{~V}$ & 4.54 & -4.14 & 7.95 & 7.42 & 0.69 & 6.96 & -3.84 & -4.68 & -8.82 \\
\hline MT336 & B3 III & 4.23 & -2.01 & 11.43 & 10.84 & 0.71 & 10.36 & -0.44 & -0.90 & -2.91 \\
\hline MT300 & B1 V & 4.38 & -3.13 & 10.71 & 10.13 & 0.74 & 9.63 & -1.17 & -1.83 & -4.96 \\
\hline MT299 & $\mathrm{O} 7 \mathrm{~V}$ & 4.57 & -4.33 & 8.19 & 7.72 & 0.63 & 7.30 & -3.50 & -4.36 & -8.69 \\
\hline MT339 & $08 \mathrm{~V}$ & 4.54 & -4.14 & 8.58 & 7.98 & 0.76 & 7.47 & -3.33 & -4.17 & -8.30 \\
\hline MT264 & B2 III & 4.26 & -2.20 & 10.48 & 10.10 & 0.56 & 9.72 & -1.08 & -1.64 & -3.84 \\
\hline MT275 & B2 V & 4.29 & -2.55 & 11.05 & 10.61 & 0.55 & 10.24 & -0.56 & -1.15 & -3.70 \\
\hline MT343 & B1 V & 4.38 & -3.13 & 10.23 & 9.36 & 1.01 & 8.68 & -2.12 & -2.78 & -5.91 \\
\hline MT358 & B3 V & 4.27 & -2.39 & 10.53 & 9.66 & 0.96 & 9.02 & -1.78 & -2.27 & -4.66 \\
\hline \#9 & O5 If & 4.59 & -4.47 & 6.47 & 5.57 & 1.09 & 4.84 & -5.96 & -6.83 & -11.29 \\
\hline MT477 & B0 V & 4.47 & -3.78 & 10.23 & 9.43 & 0.98 & 8.77 & -2.03 & -2.79 & -6.57 \\
\hline MT455 & O8 V & 4.54 & -4.14 & 9.03 & 8.28 & 0.91 & 7.67 & -3.13 & -3.97 & -8.11 \\
\hline MT448 & O6 V & 4.59 & -4.47 & 8.98 & 8.01 & 1.13 & 7.25 & -3.55 & -4.44 & -8.91 \\
\hline MT417 & O4 III & 4.63 & -4.72 & 7.11 & 6.23 & 1.08 & 5.50 & -5.30 & -6.18 & -10.90 \\
\hline MT426 & B0 V & 4.47 & -3.78 & 9.44 & 8.63 & 0.99 & 7.96 & -2.84 & -3.60 & -7.38 \\
\hline MT515 & B1 V & 4.38 & -3.13 & 10.24 & 9.36 & 1.03 & 8.67 & -2.13 & -2.79 & -5.92 \\
\hline MT480 & O7 V & 4.57 & -4.30 & 8.35 & 7.65 & 0.85 & 7.08 & -3.72 & -4.58 & -8.88 \\
\hline MT507 & O9 V & 4.52 & -3.99 & 9.30 & 8.67 & 0.81 & 8.13 & -2.67 & -3.46 & -7.45 \\
\hline MT492 & B1 V & 4.38 & -3.13 & 11.24 & 10.54 & 0.85 & 9.98 & -0.82 & -1.48 & -4.61 \\
\hline MT441 & B2 III & 4.26 & -2.20 & 11.04 & 10.39 & 0.83 & 9.83 & -0.97 & -1.53 & -3.72 \\
\hline
\end{tabular}


Table 3. continued.

\begin{tabular}{|c|c|c|c|c|c|c|c|c|c|c|}
\hline Name & $\begin{array}{c}\text { Spectral } \\
\text { type }\end{array}$ & $\log T_{\text {eff }}$ & $\overline{\overline{B . C} .}$ & $\overline{\bar{J}}$ & $\overline{\overline{K_{\mathrm{s}}}}$ & $\overline{E E(J-K)}$ & $\overline{\bar{M} M_{K}}$ & $K_{0}$ & $\overline{\overline{V_{0}}}$ & $\overline{M_{\text {bol }}}$ \\
\hline MT400 & B1 V & 4.38 & -3.13 & 10.61 & 9.94 & 0.82 & 9.39 & -1.41 & -2.07 & -5.20 \\
\hline МT390 & $\mathrm{O} 8 \mathrm{~V}$ & 4.54 & -4.14 & 8.72 & 7.87 & 1.01 & 7.20 & -3.60 & -4.44 & -8.58 \\
\hline MT395 & B1 V & 4.38 & -3.13 & 10.27 & 9.55 & 0.87 & 8.97 & -1.83 & -2.49 & -5.62 \\
\hline$\# 8 \mathrm{C}$ & O5 III & 4.61 & -4.56 & 7.17 & 6.58 & 0.79 & 6.05 & -4.75 & -5.63 & -10.19 \\
\hline \#8B & O7 II-III & 4.56 & -4.22 & 7.21 & 6.57 & 0.83 & 6.01 & -4.79 & -5.64 & -9.85 \\
\hline \#8A & O5.5 I & 4.58 & -4.38 & 6.12 & 5.50 & 0.81 & 4.96 & -5.84 & -6.71 & -11.09 \\
\hline MT473 & $08.5 \mathrm{~V}$ & 4.53 & -4.06 & 8.84 & 8.24 & 0.78 & 7.71 & -3.09 & -3.91 & -7.97 \\
\hline MT516 & $\mathrm{O} 5.5 \mathrm{~V}$ & 4.60 & -4.56 & 7.03 & 6.05 & 1.14 & 5.29 & -5.51 & -6.40 & -10.96 \\
\hline MT435 & B0 V & 4.47 & -3.78 & 10.17 & 9.28 & 1.07 & 8.57 & -2.23 & -2.99 & -6.77 \\
\hline MT378 & B0 V & 4.47 & -3.78 & 9.05 & 8.14 & 1.08 & 7.42 & -3.38 & -4.14 & -7.92 \\
\hline MT365 & B1 V & 4.38 & -3.13 & 10.79 & 10.15 & 0.78 & 9.63 & -1.17 & -1.83 & -4.96 \\
\hline MT376 & $08 \mathrm{~V}$ & 4.54 & -4.14 & 8.89 & 8.31 & 0.73 & 7.82 & -2.98 & -3.82 & -7.95 \\
\hline MT429 & B0 V & 4.47 & -3.78 & 9.54 & 8.90 & 0.82 & 8.35 & -2.45 & -3.21 & -6.99 \\
\hline MT427 & B4 II-III & 4.22 & -2.01 & 12.70 & 12.23 & 0.56 & 11.86 & 1.06 & 0.79 & -1.22 \\
\hline MT490 & B0 & 4.47 & -3.78 & 11.06 & 10.26 & 0.97 & 9.61 & -1.19 & -1.95 & -5.73 \\
\hline MT493 & B5 IV & 4.21 & -1.82 & 11.13 & 10.33 & 0.89 & 9.74 & -1.06 & -1.48 & -3.31 \\
\hline \#7 & $\mathrm{O} 3$ If & 4.63 & -4.72 & 7.25 & 6.61 & 0.83 & 6.06 & -4.74 & -5.61 & -10.33 \\
\hline MT470 & O9 V & 4.52 & -3.99 & 9.33 & 8.73 & 0.79 & 8.20 & -2.60 & -3.39 & -7.38 \\
\hline MT428 & B1 V & 4.38 & -3.13 & 10.20 & 9.44 & 0.91 & 8.84 & -1.96 & -2.62 & -5.75 \\
\hline MT409 & B $0.5 \mathrm{~V}$ & 4.40 & -3.23 & 10.39 & 9.67 & 0.88 & 9.08 & -1.72 & -2.41 & -5.64 \\
\hline MT485 & $\mathrm{O} 8 \mathrm{~V}$ & 4.54 & -4.14 & 8.74 & 8.11 & 0.79 & 7.58 & -3.22 & -4.06 & -8.19 \\
\hline MT517 & B1 V & 4.38 & -3.13 & 10.40 & 9.78 & 0.78 & 9.26 & -1.54 & -2.20 & -5.33 \\
\hline MT522 & B2 Ve? & 4.29 & -2.55 & 10.92 & 10.29 & 0.74 & 9.79 & -1.01 & -1.60 & -4.15 \\
\hline MT513 & B2 V & 4.29 & -2.55 & 10.95 & 10.32 & 0.74 & 9.82 & -0.98 & -1.57 & -4.13 \\
\hline MT469 & B1 III & 4.32 & -2.68 & 10.36 & 9.72 & 0.83 & 9.17 & -1.63 & -2.28 & -4.96 \\
\hline MT372 & B0 V & 4.47 & -3.78 & 10.51 & 9.60 & 1.09 & 8.87 & -1.93 & -2.69 & -6.47 \\
\hline MT568 & B3 V & 4.27 & -2.39 & 10.28 & 9.42 & 0.96 & 8.78 & -2.02 & -2.51 & -4.90 \\
\hline MT403 & B1 V & 4.38 & -3.13 & 9.29 & 8.62 & 0.81 & 8.08 & -2.72 & -3.38 & -6.51 \\
\hline MT425 & B0 V & 4.47 & -3.78 & 9.44 & 8.63 & 0.99 & 7.96 & -2.84 & -3.60 & -7.38 \\
\hline MT534 & $08.5 \mathrm{~V}$ & 4.53 & -4.06 & 8.97 & 8.17 & 0.99 & 7.50 & -3.30 & -4.12 & -8.18 \\
\hline MT556 & B1 I & 4.32 & -2.65 & 6.49 & 5.54 & 1.08 & 4.82 & -5.98 & -6.49 & -9.14 \\
\hline MT467 & B1 V & 4.38 & -3.13 & 10.03 & 9.34 & 0.84 & 8.78 & -2.02 & -2.68 & -5.81 \\
\hline \#10 & 09 I & 4.50 & -3.76 & 6.29 & 5.58 & 0.87 & 5.00 & -5.80 & -6.53 & -10.29 \\
\hline MT531 & $08.5 \mathrm{~V}$ & 4.53 & -4.06 & 8.17 & 7.52 & 0.83 & 6.97 & -3.83 & -4.65 & -8.71 \\
\hline MT611 & $07 \mathrm{~V}$ & 4.57 & -4.30 & 9.26 & 8.61 & 0.80 & 8.08 & -2.72 & -3.58 & -7.88 \\
\hline MT509 & B0 III-IV & 4.48 & -3.68 & 10.22 & 9.39 & 1.01 & 8.71 & -2.09 & -2.78 & -6.46 \\
\hline MT555 & $\mathrm{O} 8 \mathrm{~V}$ & 4.54 & -4.14 & 8.39 & 7.57 & 0.98 & 6.91 & -3.89 & -4.73 & -8.86 \\
\hline MT531 & $08.5 \mathrm{~V}$ & 4.53 & -4.06 & 8.17 & 7.52 & 0.83 & 6.97 & -3.83 & -4.65 & -8.71 \\
\hline MT601 & B0 Iab & 4.46 & -3.60 & 7.23 & 6.48 & 0.89 & 5.89 & -4.91 & -5.50 & -9.10 \\
\hline MT573 & B3 I & 4.21 & -1.80 & 10.27 & 9.42 & 0.94 & 8.79 & -2.01 & -2.35 & -4.15 \\
\hline MT575 & B2 Ve & 4.29 & -2.55 & 9.10 & 7.85 & 1.36 & 6.94 & -3.86 & -4.45 & -7.01 \\
\hline MT642 & B1 III & 4.32 & -2.68 & 7.99 & 7.21 & 0.97 & 6.56 & -4.24 & -4.89 & -7.57 \\
\hline MT646 & $\mathrm{B} 1.5 \mathrm{~V}$ & 4.30 & -2.55 & 10.03 & 9.37 & 0.78 & 8.85 & -1.95 & -2.57 & -5.13 \\
\hline MT696 & O9.5 V & 4.50 & -3.91 & 8.53 & 7.89 & 0.82 & 7.34 & -3.46 & -4.23 & -8.13 \\
\hline MT605 & $\mathrm{B} 1 \mathrm{~V}$ & 4.38 & -3.13 & 8.88 & 8.28 & 0.75 & 7.78 & -3.02 & -3.68 & -6.81 \\
\hline MT561 & B2 V & 4.29 & -2.55 & 10.85 & 10.16 & 0.80 & 9.62 & -1.18 & -1.77 & -4.32 \\
\hline MT588 & B0 V & 4.47 & -3.78 & 8.68 & 7.93 & 0.93 & 7.30 & -3.50 & -4.26 & -8.04 \\
\hline MT620 & B0 V & 4.47 & -3.78 & 9.89 & 9.10 & 0.97 & 8.45 & -2.35 & -3.11 & -6.89 \\
\hline MT645 & B2 III & 4.26 & -2.20 & 10.58 & 9.78 & 0.98 & 9.12 & -1.68 & -2.24 & -4.43 \\
\hline MT712 & B1 V & 4.38 & -3.13 & 9.69 & 8.89 & 0.95 & 8.25 & -2.55 & -3.21 & -6.34 \\
\hline MT720 & $09.5 \mathrm{~V}$ & 4.50 & -3.91 & 9.05 & 8.15 & 1.06 & 7.44 & -3.36 & -4.13 & -8.04 \\
\hline MT692 & B0 V & 4.47 & -3.78 & 9.99 & 9.30 & 0.87 & 8.72 & -2.08 & -2.84 & -6.62 \\
\hline MT621 & B1 V? & 4.38 & -3.13 & 10.74 & 9.89 & 1.00 & 9.22 & -1.58 & -2.24 & -5.37 \\
\hline MT635 & B1 III & 4.32 & -2.68 & 10.16 & 9.40 & 0.95 & 8.77 & -2.03 & -2.68 & -5.36 \\
\hline МT639 & B2 V & 4.29 & -2.55 & 10.50 & 9.71 & 0.89 & 9.11 & -1.69 & -2.28 & -4.83 \\
\hline MT716 & O9 V & 4.52 & -3.99 & 9.56 & 8.84 & 0.91 & 8.23 & -2.57 & -3.36 & -7.35 \\
\hline MT650 & $\mathrm{B} 2 \mathrm{Ve} ?$ & 4.29 & -2.55 & 11.07 & 10.33 & 0.85 & 9.76 & -1.04 & -1.63 & -4.18 \\
\hline \#11 & O5 I & 4.59 & -4.47 & 6.65 & 5.99 & 0.85 & 5.42 & -5.38 & -6.25 & -10.72 \\
\hline MT745 & $\mathrm{O} 7 \mathrm{~V}$ & 4.57 & -4.30 & 8.55 & 7.92 & 0.78 & 7.40 & -3.40 & -4.26 & -8.56 \\
\hline MT736 & O9 V & 4.52 & -3.99 & 9.30 & 8.65 & 0.84 & 8.08 & -2.72 & -3.51 & -7.49 \\
\hline MT759 & B1 V & 4.38 & -3.13 & 10.87 & 10.11 & 0.91 & 9.50 & -1.30 & -1.96 & -5.09 \\
\hline MT793 & B2 IIIJe & 4.26 & -2.20 & 8.61 & 7.70 & 1.09 & 6.97 & -3.83 & -4.39 & -6.59 \\
\hline MT771 & $\mathrm{O} 7 \mathrm{~V}$ & 4.57 & -4.30 & 7.56 & 6.71 & 1.00 & 6.04 & -4.76 & -5.62 & -9.92 \\
\hline
\end{tabular}


I. Negueruela et al.: New very massive stars in Cygnus OB2, Online Material p 4

Table 3. continued.

\begin{tabular}{lclllllllll}
\hline \hline Name & $\begin{array}{c}\text { Spectral } \\
\text { type }\end{array}$ & $\log T_{\text {eff }}$ & B.C. & $J$ & $K_{\mathrm{s}}$ & $E(J-K)$ & $M_{K}$ & $K_{0}$ & $V_{0}$ & $M_{\text {bol }}$ \\
\hline A11 & O7.5 Ibf & 4.53 & -4.06 & 7.82 & 6.66 & 1.32 & 5.78 & -5.02 & -5.84 & -9.90 \\
A24 & O6.5 IIIf & 4.57 & -4.38 & 8.41 & 7.45 & 1.15 & 6.68 & -4.12 & -4.98 & -9.36 \\
A38 & O8 V & 4.54 & -4.14 & 9.38 & 8.56 & 0.98 & 7.91 & -2.89 & -3.73 & -7.87 \\
A33 & B0.2 V & 4.45 & -3.59 & 9.44 & 8.61 & 1.01 & 7.93 & -2.87 & -3.60 & -7.19 \\
A37 & O5 V & 4.61 & -4.64 & 8.57 & 7.69 & 1.04 & 6.99 & -3.81 & -4.70 & -9.35 \\
A32 & O9.5 IV & 4.50 & -3.91 & 7.89 & 7.07 & 1.01 & 6.39 & -4.41 & -5.15 & -9.06 \\
A29 & O9.7Iab & 4.48 & -3.68 & 7.44 & 6.55 & 1.05 & 5.84 & -4.96 & -5.58 & -9.26 \\
A27 & B0 Ia & 4.46 & -3.60 & 6.68 & 5.73 & 1.14 & 4.97 & -5.83 & -6.47 & -10.07 \\
A23 & B0.7 Ib & 4.33 & -2.65 & 6.93 & 5.98 & 1.08 & 5.26 & -5.54 & -6.06 & -8.71 \\
A20 & O8I If & 4.52 & -3.99 & 7.25 & 6.27 & 1.16 & 5.50 & -5.30 & -6.11 & -10.10 \\
A46 & O7 Vf & 4.57 & -4.30 & 8.38 & 7.83 & 0.70 & 7.36 & -3.44 & -4.30 & -8.60 \\
A45 & B0.5 V(n)sb2? & 4.40 & -3.25 & 9.03 & 8.46 & 0.74 & 7.96 & -2.84 & -3.53 & -6.78 \\
A44 & B0.5 IV & 4.40 & -3.25 & 8.91 & 8.26 & 0.84 & 7.69 & -3.11 & -3.78 & -7.03 \\
A42 & B0 V & 4.47 & -3.78 & 9.12 & 8.45 & 0.85 & 7.88 & -2.92 & -3.68 & -7.45 \\
A41 & O9.7 II & 4.48 & -3.68 & 7.83 & 7.02 & 0.96 & 6.38 & -4.42 & -5.04 & -8.72 \\
A39 & B2 V & 4.29 & -2.55 & 8.62 & 7.88 & 0.85 & 7.31 & -3.49 & -4.08 & -6.64 \\
A37 & O5 Vf & 4.61 & -4.64 & 8.57 & 7.69 & 1.04 & 6.99 & -3.81 & -4.70 & -9.35 \\
A36 & B0 Ib(n)sb2? & 4.46 & -3.60 & 7.19 & 6.36 & 0.98 & 5.70 & -5.10 & -5.69 & -9.29 \\
A34 & B0.7 Ib & 4.33 & -2.65 & 7.36 & 6.66 & 0.83 & 6.10 & -4.70 & -5.22 & -7.87 \\
A12 & B0 Ia & 4.46 & -3.60 & 6.93 & 5.72 & 1.40 & 4.79 & -6.01 & -6.65 & -10.25 \\
A15 & O7 Ibf & 4.54 & -3.31 & 7.94 & 6.81 & 1.32 & 5.92 & -4.88 & -5.72 & -9.03 \\
A26 & O9.5 V & 4.50 & -3.91 & 9.16 & 8.19 & 1.14 & 7.42 & -3.38 & -4.15 & -8.06 \\
A18 & O8 V & 4.53 & -3.23 & 9.41 & 8.35 & 1.25 & 7.51 & -3.29 & -4.09 & -7.32 \\
A25 & O8 III & 4.53 & -3.23 & 8.37 & 7.36 & 1.19 & 6.56 & -4.24 & -5.04 & -8.27 \\
\hline
\end{tabular}

